\title{
Experience of operation of the gas turbine units in Russky Island
}

\author{
Dmitry Bibikov ${ }^{1,{ }^{*}}$, Anton Polei ${ }^{1}$, Konstantin Shtym ${ }^{1}$, and Konstantin Tsoi ${ }^{1}$ \\ ${ }^{1}$ Far-Eastern Federal University, 8, Sukhanova Street, Vladivostok 690950
}

\begin{abstract}
In the present article, the heat and power supply system based on the distributed cogeneration and created as part of the APEC Summit and development of the southern Far East is considered. A brief review of the experience of operating the gas turbine units of the Co-generation plant in the Russky Island is given. The output of the heat energy over 2015 reached 142.228 thousands Gcal which is larger than that in 2014 by 18.8 $\%$. The specific reference fuel consumption for electric supply was 272.4 $\mathrm{g} /\left(\mathrm{kW}^{*} \mathrm{~h}\right)$ while for heat supply $-195.8 \mathrm{~kg} / \mathrm{Gcal}$. In spite of partial load of the stations with regard to electric and heat energy (volumes of consumptions by the connected consumers is less than designed loads), the indices of reference fuel consumption suggest the efficiency of the generation equipment of co-generation plant.
\end{abstract}

\section{Introduction}

The development of the Russky Island infrastructure for the 2012 APEC Summit in Vladivostok has begun from the construction of electric-power and heat-power facilities. From the inland Vladivostok, the cable of $220 \mathrm{kV}$ and gas pipeline for the fuel supply of three co-generation plants ("North" $(\mathrm{Ne}=5.6 \mathrm{MW}, \mathrm{Nh}=12.4 \mathrm{MW})$, ("Central" $(\mathrm{Ne}=33$ $\mathrm{MW}, \mathrm{Nh}=143.4 \mathrm{MW})$ and "Oceanarium" $(\mathrm{Ne}=13.2 \mathrm{MW}, \mathrm{Nh}=34.3 \mathrm{MW})$ were laid through the Bosfor-Vostochny strait. The basic equipment of the co-generation plant includes the water-heating fire-tube boilers and gas turbine units (GTU) with waste heat recovery units.

\section{Basic part of report}

The used GTU was GPB-70D with synchronous, containerized module generator GFV8 made by «Fuji Electric» firm with nominal power of 6.6 MW when operated at gas and 5.8 MW when operated at diesel fuel (at an ambient temperature of $10^{\circ} \mathrm{C}$ ). The gas turbine installation (Fig.1) was made in the single-shaft arrangement with axial once-through medium circulation operating according to Brayton thermodynamic cycle.

The compressor of GTU has 11 steps, and first three compressor blades are equipped with swing function by means of the leverage mechanism and electrodrive. At GTU starting, these blades provide a minimum angle in order to lower starting power and to

*Corresponding author: dibibikov@yandex.ru 
avoid the compressor swinging. At the outlet section of the compressor, near the combustion can, the air outlet port is mounted to open at a startup for reducing pressure in the compressor. The coefficient of pressure in compressor is 1:16 at air flow rate of 26.7 $\mathrm{kg} / \mathrm{s}$. A part of compressed air at the output is used after passing through the rotor for cooling the turbine first stage. After the compressor fifth stage, air is discharged to the bearing housings for compaction of the lubricating oil. At the moment of start and runout of turbine, the sealing air under the pressure of $0.3 \mathrm{MPa}$ is drawn in from the compressor plants located in the engine room.

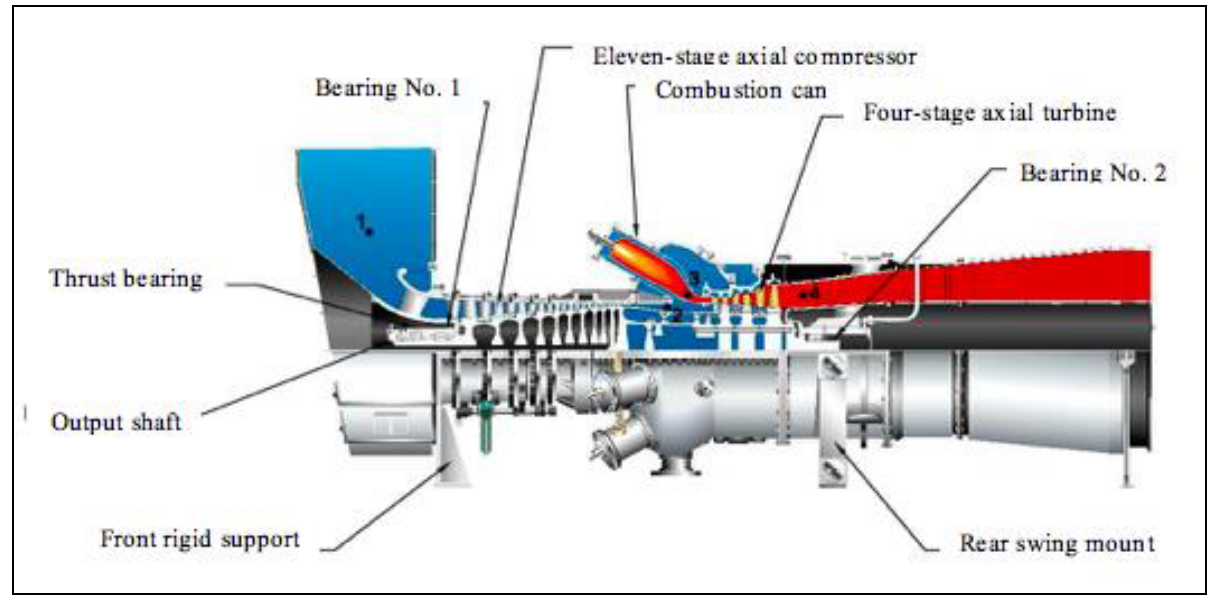

Fig.1. Longitudinal section of GTU Kawasaki GPB 70D

The natural gas pressure for operation of the installation should be supported at a level of $2.1 \mathrm{Mpa}(+-0.1 \mathrm{MPa})$, therefore to increase of the main gas pressure $(1.2 \mathrm{MPa})$, the piston, gas booster compressor VWFD-5,1/8-23 (LTB) made by Bengbu Hongshen firm (China) is used. The maximum discharge of natural gas per one GTU is $3000 \mathrm{NCMH}$.

The turbine driving a shaft consists of four stages with input temperature of $1200^{\circ} \mathrm{C}$; the first stage is cooled by the compressor-bleed air и and made of high-temperature resistant metal with thermal barrier coating. The generator shaft is connected with the turbine through couplings and planetary transmission of gear-reduction box, reducing the rotating speed from $13790 \mathrm{rpm}$ to $1500 \mathrm{rpm}$ which is also serves as a driving actuator for main oil pump and diesel fuel pump.

The combustion cans (CC) (Fig.2) have 4 injectors for gaseous fuel: control (P), primary (M1), secondary (M2), auxilliary (S) as well as 2 injectors for the fluid fuel: primary fluid (Pr), main fluid (M), at that, the control, primary, primary fluid and main fluid injectors form one fuel tube.

The presence of the secondary and auxilliary injectors allows operating in DLE (Dry Low Emission) mode, to burn the diluted premixed air mixture, used while working on natural gas in parallel with network and at load over $50 \%$.

The startup of GTU and its operation at starting modes are realized using the normal diffusion flame from the control injector (Fig. 2) in the range of «pilot zone», and burning is performed with a large excess of air $(\alpha=3.5)$. Beginning with $50 \%$ of nominal power, the fuel starts passing through the swirler and, in the range of $60-70 \%$, the injectors switch over, the control injector is shut down and, instead of it, the primary, secondary and auxilliary injectors provide burning. Hence, burning is divided into the «pilot» and «basic» zones. When the installation operates in the DLE mode, a level of NOx does not exceed 50 $\mathrm{mg} / \mathrm{m}^{3}$ which corresponds to GOST 23290-78. 


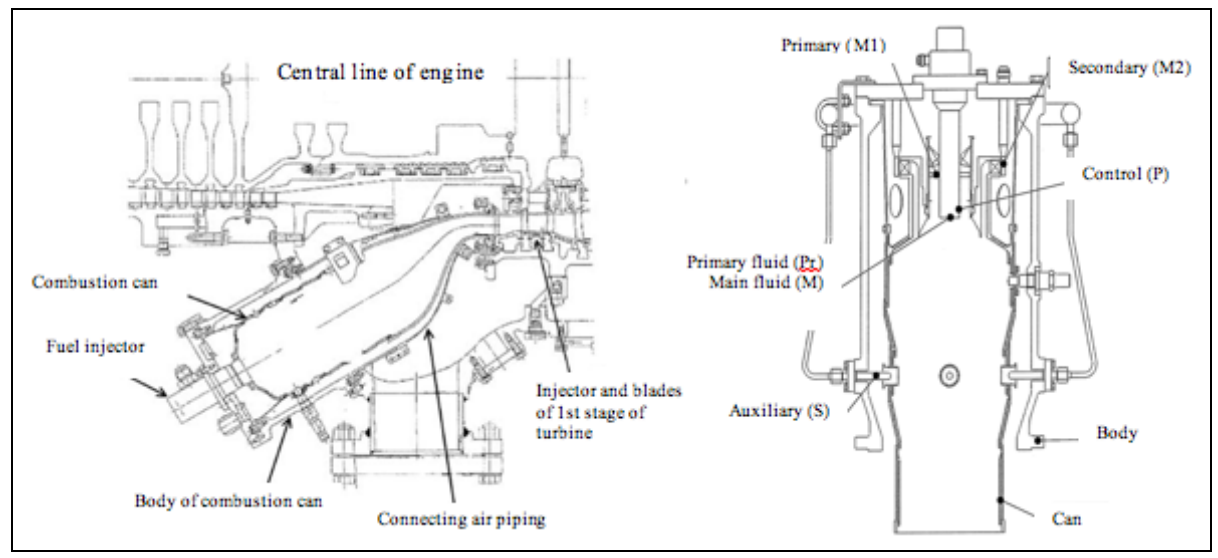

Fig. 2. Combustion can of GTU Kawasaki GPB 70D (in section)

After mounting and checking the assembly diagrams, the commissioning works with adjustment of GTU (natural gas) operating modes were accomplished (Fig. 3). In Fig. 3 shows such parameters as efficiency factor, power, mass flow of exhaust gases, flue-gas temperature, consumption of fuel depending on the ambient air temperature.

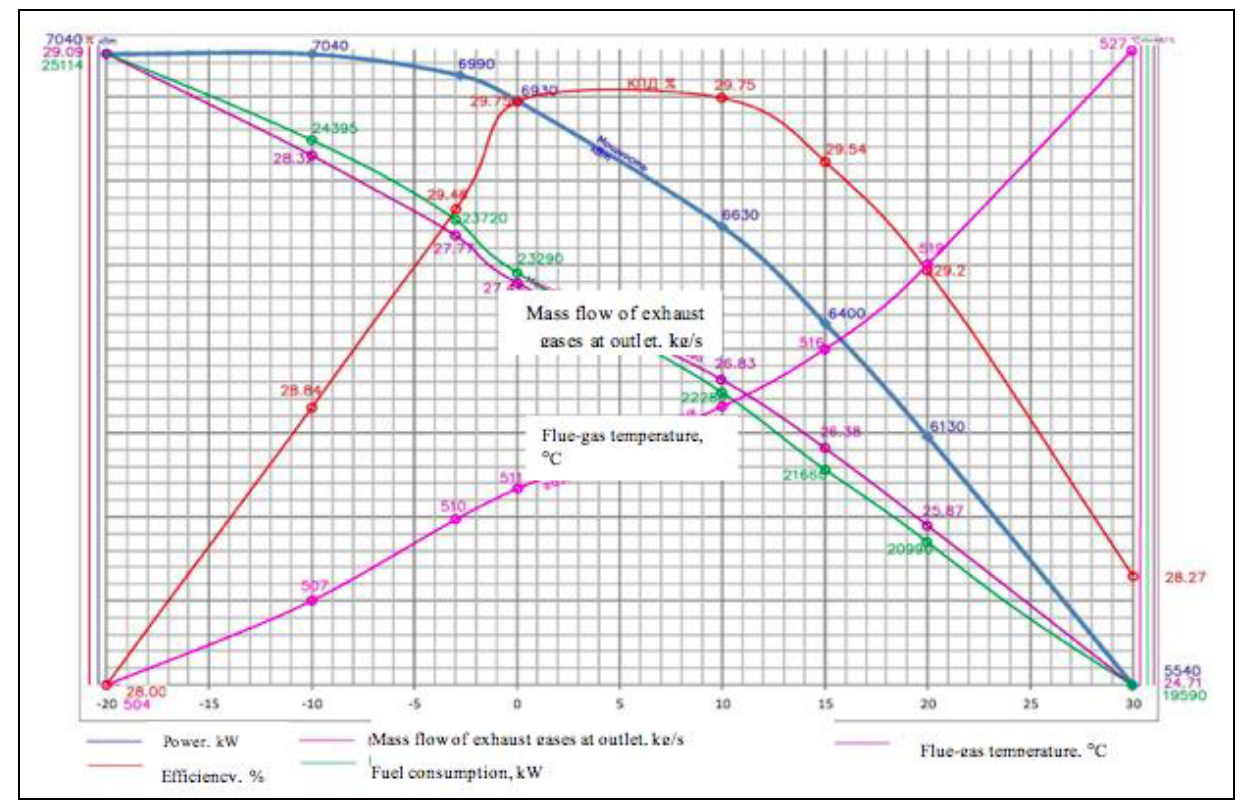

Fig. 3. Dependence of maximum power on initial temperature of GTU No/ 1 (gas) of co-generation plant «Oceanarium»

From the diagram, it follows that when increasing the ambient air temperature, the power of GTU decreases from 7040 to $5540 \mathrm{~kW}$ due to the growth of flue-gas temperature and a decrease the absolute thermal efficiency of GTU is also observed. The GTUs Kawasaki GPB 70D proved themselves as the highly automated and reliable under all operation conditions. This is really very high level of power production technology to which it is necessary to reach after.

In February, 2016, the boroscopic inspection of the combustion cans (CC) of the GTU No. 4 of the co-generation plant «Central» was carried out which showed the burning through of the deflector body (about $70 \%$ of surface area) and burn-off of edges of 
materials in the first section at two points with dimensions of 100x15mm and 40x10mm in combustion can No. 2.

The inspection of the dismounted combustion cans No. 2 and 4 (Fig. 4) was performed. The transition ducts, nozzle diaphragm blades of the first and second stages and impeller blades of the first stage of turbine were examined through the flanges of dismounted combustion cans. In two sections of the combustion can No. 4, there are segments with detachment of fireproof enamel which can result in burnings-out in case of further operation. During the following boroscopic inspections, it is needed to monitor the possible development of defect.

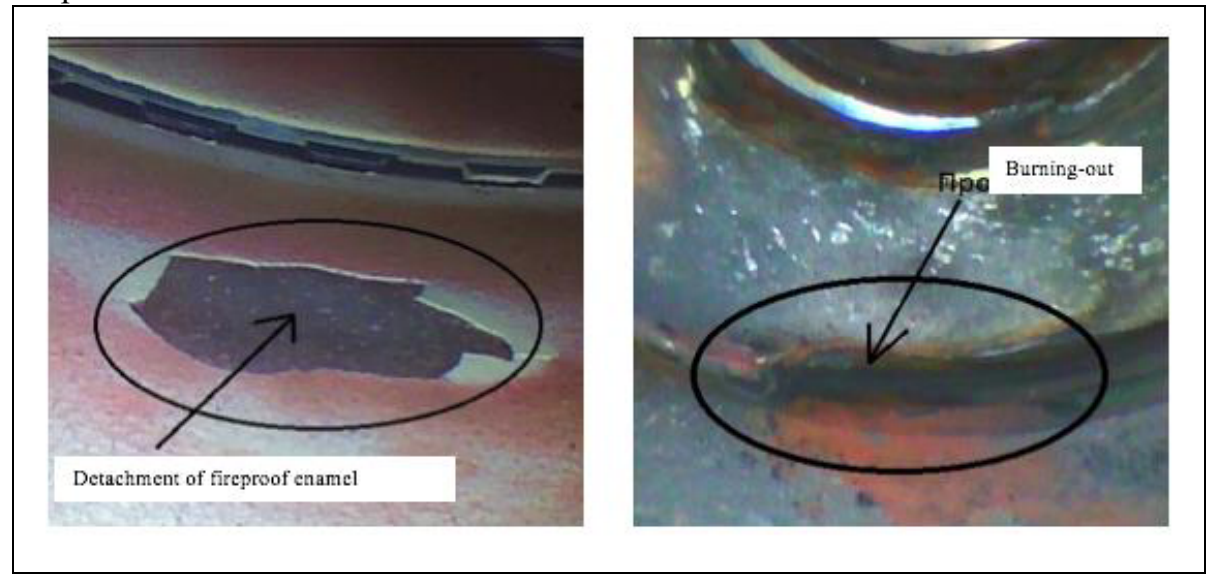

Fig. 4. Combustion can No. 2 and 4

\section{Conclusion}

The installed equipment of the distributed energetics based on co-generation is the first experience in the Far-Eastern region and holds great prospects for development. Their reliability and efficiency will serve to provide consumers of the Russky Island with heat and electric energy. In 2016, mean life time of machines reached 3500 after which the boroscopic inspection of all machines was carried out and the CCs and nozzle blocks were subject to the non-destructive testing. As of April, 2017, the mean time of the GTU of cogeneration plant is 7000 equivalent hours, it means that the following stage of maintenance (inspection of high-temperature part which will show the state of transition duct and blades of turbine) is needed in order to timely determine and eliminate defects which can arise in operation.

\section{References}

1. Kawasaki GPB70D. Generator plant of gas turbine. Operation manual. Kawasaki Heavy Industries, LTD. (to be published)

2. S.V. Tsanev, V.D. Burov, A.N. Relizov, Gas-turbine and combined cycle installations of power plants $\mathbf{5 8 4}$ (2002)

3. Y.B. Goncharenko, A.K. Polei, SB of RAS 225 (2014)

4. K.A. Tsoi, A.K. Polei, M.A. Makarov, Collection of Scientific Papers 646 (2016) 